\title{
IMPLEMENTING A COMMON APPROACH TO PROBLEM SOLVING IN THE SECOND YEAR OF CHEMICAL ENGINEERING
}

\author{
Louise Meunier and Nicolas Hudon \\ Chemical Engineering Department, Queen's University, Canada \\ louise.meunier@queensu.ca
}

\begin{abstract}
At the undergraduate level, chemical engineering students must learn how to solve complex problems, but many students fail to apply effective problem-solving techniques taught in fundamental science courses. Because these techniques are not consistently presented and reinforced, instructors and students often interpret poor capabilities in problem solving to misunderstandings of fundamentals or to gaps in mathematical knowledge. In this contribution, a previously proposed concept map aimed at ascribing a common approach to problem solving is further explored in a sequence of two junior-level chemical engineering courses. The difficulties of implementing a common problemsolving approach are discussed, and a hierarchy of problem solving is proposed - based on a combination of learning theories - to structure a problem-solving methodology from junior to senior level as well as toward graduate studies and professional practice. Preliminary results indicate that students benefiting from this structured approach exhibit improved confidence in their problem-solving abilities. The proposed concept map forms the basis of future stages of the project, including curriculum and teaching innovations.
\end{abstract}

Keywords: Problem-solving Skills; Solution Procedure; Chemical Engineering; Junior Level; Curriculum Alignment; Fundamentals of Mass and Energy Balances; Constructivism Theory; Objectivism Learning Theory; Situated Design Cognition; Problem-Based Learning Theory.

\section{INTRODUCTION}

Many junior-level courses in undergraduate chemical engineering require students to solve problem of increasing difficulty. The success of each student depends on their ability to thoroughly understand and to apply both the fundamental concepts learned thus far in their studies and the methodology required to systematically solve these problems.

\subsection{Motivation}

In this contribution, we outline a project aiming to adopt a common approach to problem solving across the secondyear chemical engineering curriculum. The goal of this concerted effort is to improve student abilities to solve problems and to increase their confidence in tackling problems of increasing complexity. The project involves a sequence of two fundamental courses over two semesters, and the project is currently in its second year of data collection. Data is also collected from students currently in their third year of studies and who have experienced this common approach during their second year. The curriculum-wide approach to problem solving will be adjusted depending on their responses. This represents a novel approach in decompartmentalizing how students learn by adapting the curriculum delivery retroactively and proactively. At this point in the investigation, the project involves two courses in which teaching methods and philosophy are aligned. Thus, a consistent problem-solving approach highlights the similarities in concepts learned through different courses. The vision for this project is to expand the proposed consistent problem-solving methodology to the entire chemical engineering undergraduate curriculum.

\subsection{Literature Review}

In a first contribution produced on this topic [1], the authors summarized the development of problem-solving theories, and described the steps required in problem solving, from the perspective of the problem-based learning theory. They posited that the difficulty in solving a problem is often associated with interpreting relevant information into an actionable solution procedure. Fundamentally, students may possess the knowledge required to solve a problem but lack the ability to relate the problem information to this knowledge. Therefore, the application of effective problem-solving techniques must be framed beyond the organization of information and the use of solution procedure to a broader understanding of how students learn.

To illustrate how engineering-undergraduate students acquire and integrate knowledge, three theories of learning 
are discussed: constructivism, objectivism, and situated design cognition. According to the constructivism learning theory, learners build knowledge by actively incorporating new information into a pre-existing knowledge structure [2]. The act of learning requires that the learner take in the new information which is presented to them through experiences and interactions. The instructor's role is to propose a scaffolding mechanism for the new information to be taken up by the student. However, such knowledge incorporation must be woven into prior experiences and existing knowledge, which always influence the formation of new knowledge, and requires that the learner actively participates in the process [3]. In contrast, objectivists view the body of human knowledge as a tangible and reliable set of facts, principles, and theories; these exist objectively, externally, and independent from the learner [4]. Objectivistic instructors view the effective transmission of information as pivotal. The instructor presents what must be learned as clearly as possible, and students are responsible for absorbing this information as a pure set of facts [5]. Constructivism and objectivism may appear mutually exclusive - even mutually destructive - but Cronjé [6] submitted that they are complementary: they may be plotted at right angles to each other, thereby allowing a learning event to synergistically present constructivist and objectivist features. The degree of integration of these two learning theories may be further illustrated by dividing this plot into four quadrants, which the author labels as injection, immersion, construction, and integration. Injection is characterized by high objectivism and low constructivism, whereby students learn established procedures through predetermined examples and practice. This is most often how junior-level engineering courses are presented. The immersion quadrant of low objectivism and low constructivism presents information chaotically and the student is left to sort out learning opportunities without much guidance. In such scenarios, learning is not planned and often results from mistakes. In contrast, the construction quadrant features a low objectivist and highly constructivist learning environment, which favours the development of assumptions and individual understanding through exploration: the student seeks useful knowledge by looking for needed information with minimal guidance from the instructor beyond the presentation of facts. Undergraduate engineering students must strive to develop these abilities, although it may be difficult to achieve a truly independent level of understanding without the benefit of years of practice and experience. Integration, the last quadrant, encapsulates high objectivist and high constructivist learning elements. This approach is suitable when students have acquired previous experience and have moved beyond introductory level in their knowledge base. Instruction is then designed to meet specific goals through knowledge integration and appropriate practice.

In the case of engineering undergraduate students, who aspire to apply scientific knowledge as part of their professional work, the effective implementation of an integrated objectivist and constructivist approach requires that knowledge acquisition take place through realistic scenarios. Student require much practice and exposure to various engineering applications to develop an understanding of what constitutes a sound set of parameters. This realization is embodied in the situated cognition theory: learning is inseparable from doing [7]. In his seminal publication Experience and Education, Dewey [8] suggested that both objective and internal conditions form a learning situation. Objectively, the purpose of education is to prepare a student for future responsibilities. This is achieved by exposing students to organized bodies of information, communicated by teachers who act as agents of knowledge and skills. Intrinsic motivation and acquired learning behaviours allow students to internalize this knowledge. In this light, a cognitive learning activity in an engineering class must guide the learner to emulate a professional [7]. Given a specific scenario, students are invited to utilize their knowledge and to think about how a professional engineer would tackle the problem. A student most not only be capable of solving the problem but must also be capable of assessing the validity of the solution.

\subsection{Problem Definition}

At the beginning of their second year of undergraduate studies in chemical engineering, students have acquired, or at least have been exposed to, most of the necessary mathematical and fundamental scientific knowledge. They have thus far been successful in their studies, but this success has typically been achieved through a hodgepodge of methods and learning strategies. As they progress through their engineering studies, the knowledge they must acquire and the problems they are asked to solve become increasingly difficult and complex. Haphazard approaches are no longer enough, and many students face significant obstacles in achieving the expected solutions. Therefore, a systematic problem-solving methodology becomes paramount. As previously hypothesized [1], students find it especially difficult to formulate actionable solution procedures upon reading a typical chemical engineering problem. In this paper, we focus on the tangible steps required to overcome the difficulties of adopting a curriculum-wide problem-solving approach, as well as teaching activities and approaches exploited in the last two academic years to foster problem-solving capabilities in second-year chemical engineering students. This contribution also discusses how survey results were considered in the improvement of the proposed effort.

\subsection{Selected Methodologies}

In the past two academic years, instructors involved in a sequence of two fundamental courses of the second-year undergraduate chemical engineering program have coordinated their teaching approach. The first course in 
chemical processes and systems (Course A) is delivered during the fall term, and the second course in thermodynamics of energy conversion systems (Course B) follows in the winter term. Both courses are aligned to present all problems from the perspective of mass, energy, and entropy balances, as appropriate.

1.4.1. Data Collection. At the beginning and end of each term, students are invited to fill out a survey on their perceived confidence and abilities to solve problems. In addition, informal feedback was sought during each term, and students filled out course evaluations at the end of each term. Instructors adjusted their teaching strategies based on these combined responses.

1.4.2. Pedagogical Approach. During the first year of implementation, Course A instructor emphasized the implementation of a detailed problem-solving methodology, which was applied throughout all in-class examples, tutorials, practice problems, assignments, and examinations. Generally, students who applied the proposed solution method developed the desired problemsolving habits and improved their abilities and confidence in problem solving. However, nearly $20 \%$ of the class obtained a failing grade in Course A, which is in line with the historical performance of students in this course.

Course B followed essentially the same procedures. Historically, Course B instructor delivered the material and presented a problem-solving approach following ideas from Wankat and Oreovicz [9] and Polya [10]. From this pedagogical perspective, emphasis was placed on the preliminary steps of problem-solving, i.e., understanding the problem and translating the problem into actionable steps. Yet, early survey results demonstrated that the main difficulty perceived by students was to establish the mathematical framework. In response, the instructor of Course B gradually shifted the problem-solving demonstrations to explicitly illustrate the mathematical contents, which led to the development of a concept map centered on the balance equations.

During the second year of implementation, instructors presented the same problem-solving methodology, but also described the theoretical framework to raise student awareness of learning strategies and the thought processes involved as they work through each problem. In addition to the methods and tools previously described, students used an interactive version of the textbook (Course A) and video of problem solutions (primarily in Course B) were offered to supplement written solutions.

Instructors focused on confidence building and on further developing problem-solving and exam-preparation habits. Practice problems and assignments were especially designed for students to apply a complete and systematic mathematical framework to solve problems both analytically and with the use of a mathematical computing software.
Results showed improvements in student abilities to solve problems. During the second year of implementation, the same approach was repeated in both courses, with further emphasis on linking theoretical contents into concrete problem-solving steps. Students were also invited to actively reflect on how their problem-solving approach has evolved during their second year of engineering studies, with the clear goal for the students to gradually develop their own problem-solving approach to be carried on in upper-year engineering classes.

\subsection{Significance of the Results}

Engineers develop their problem-solving abilities throughout their career. Engineering instructors therefore have far more problem-solving experience than their students, which often makes it difficult to remember what their students do not know. The present study contributes to an increased awareness among instructors of the difficulties faced by undergraduate students in acquiring such abilities and experience. The results of this study are meant to provide instructors with evidence of more and less successful aspects of their pedagogical approaches. The long-term objective is to propose a consistent methodology applicable to all fundamental courses in the chemical engineering curriculum. The vision is to provide students with a consistent and coherent learning process that promotes a comprehensive problem-solving methodology. To this end, the concept map previously proposed [1] is expanded and supported by a combination of constructivism, objectivism, and situated design cognition theories. Results of the past two years of implementation are discussed, along with an analysis of teaching effectiveness. Finally, future avenues of investigation are proposed to expand the proposed approach to other aspects of the chemical engineering curriculum.

\section{DEVELOPING A COMMON APPROACH TO PROBLEM SOLVING}

\subsection{Theoretical Framework}

Expanding on the two-dimensional plot of constructivism and objectivism proposed by Cronjé [6], a three-dimensional structure is offered to illustrate the incorporation of knowledge toward the development of problem-solving abilities. Essentially, such a construct combines the approaches of Cronjé [6] and Jenlink [7]. This framework is illustrated in Figure 1, where each axis represents a learning theory: objectivism, constructivism, and situated design cognition.

Students are exposed to facts and information (i.e. objectivism); they may acquire and integrate these into useful knowledge depending on their experiences and exposure to various problems and case studies (i.e. situated design cognition); as a result of this exposure, and guided by their instructors, students build knowledge structures 
(i.e. constructivism), which become larger and more complex as their studies and experiences proceed. During their studies and as they discover new knowledge (illustrated by the horizontal rectangular bloc in Figure 1), students must deconstruct, modify, insert new components, and rebuild their knowledge structure. Such intellectual activities are not accomplished without effort, and students must be willing to take part in this process. Once integrated, the new knowledge is assimilated into the construction; it can then interact with other knowledge and be drawn upon when required in the solution of future problems.

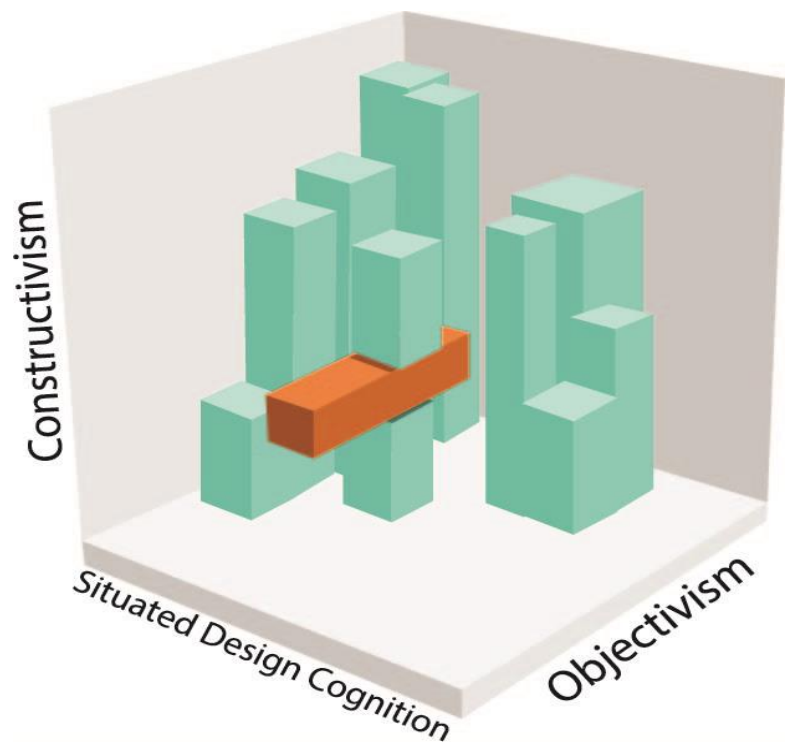

Figure 1: Knowledge Construct

Acquired knowledge is built based on facts and experience of specific situations (i.e., vertical teal blocks). These constructions allow for the retrieval and interactions of knowledge. A block of "new" concepts (i.e., horizontal orange block) may be disruptive because it must be incorporated into an existing structure of knowledge.

\subsection{Applications of a Concept Map}

The concept map introduced in a previous publication [1] is reproduced here for discussion purposes and to expand on the proposed framework. Students benefit from a consistent approach to problem solving, as demonstrated by the preliminary results obtained following the first year of curriculum alignment for Courses A \& B. The proposed concept is based on the fundamental realization that all chemical engineering courses require the application (and solution) of balance and conservation laws. Thus, the elaboration of a solution requires a consistent problemsolving methodology through which known quantities are identified (Data), in specific and realistic scenarios where simplifications may apply (Assumptions). Students then draw on their knowledge construct to identify relevant theory, formulae, and equations (Theory), and organize problem information into a collective, which is required to equilibrate mass, momentum, energy, and/or entropy. The resulting formulae express the appropriate forms of conservation equations (Balances), which lead to the answers sought (Solution Procedure), and through which students may extrapolate information that will be applied to future - and most likely more complex - problems.

A plethora of literature describes similar problemsolving methodology $[9,10,11,12]$; its successful implementation hinges on consistent, detailed, and pertinent application in the classroom and throughout the learning experience. Woods [12] remarked that students find it difficult to connect acquired knowledge applied to past problems with the current problem to be solved. Reinforcing these links through consistent approaches to problem solving can improve student confidence and abilities. However, the problem-solving methodology cannot be followed blindly; students must also develop skills in troubleshooting and recognizing wrong turns to correct mistakes along the way.

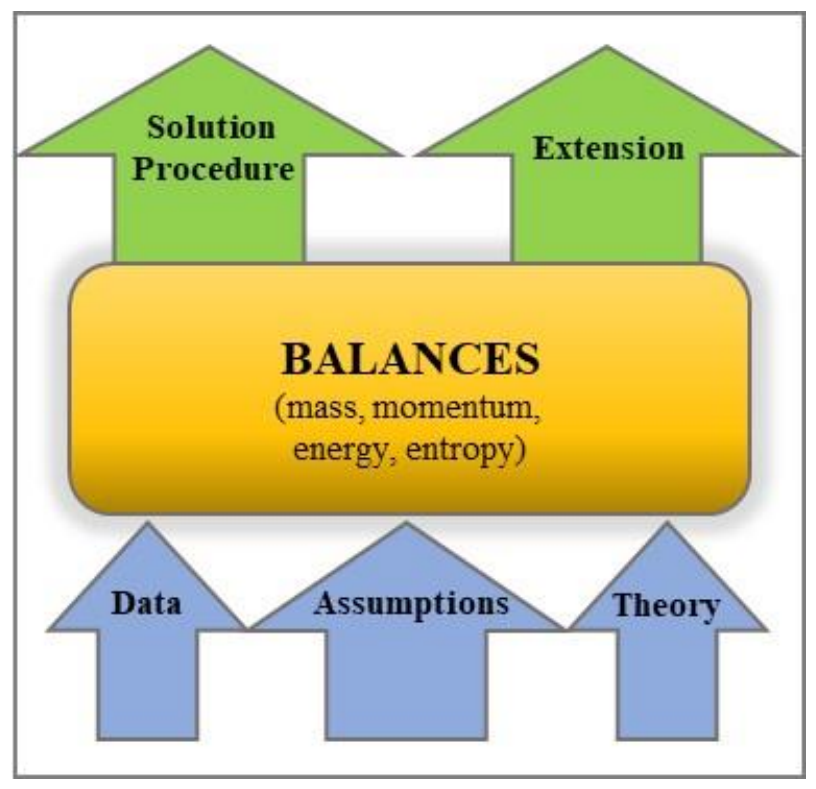

Figure 2: Concept Map

Curriculum in two consecutive courses is aligned such that all concepts are presented through the lens of conservation laws. Examples and problems are solved using a consistent methodology emphasizing similarities between all balance equations.

\subsection{Classroom Activities}

A detailed description of activities carried out in Courses A and B was previously presented [1]. Briefly, Course A material is presented in a series of modules, starting from simple mass balances at steady state, then adding variations in energy and system fluctuations over time. In Course B, the first and second laws of thermodynamics are attached to the concept of balances, notably by adding the entropy balance. Course B is then 
based on the solution of systems of balance equations to which fundamental notions on constitutive relationships and degrees of freedom analysis are gradually incorporated. This allows the students to derive solutions for systems of increasing complexity, both from a mathematical perspective and a fundamental knowledge perspective.

The problem-solving methodology is systematically applied in all aspects of each course (in-class examples, tutorials, assignments, online interactive exercises, and video examples). Special emphasis is also placed on student awareness of the process of accessing acquired knowledge to solve the current problem. As reported in this work, survey results from the first year of the project clearly demonstrated that "developing mathematical equations" is perceived by students as the most difficult step in problem solving. This prompted Courses A and B instructors to shift their teaching focus from demonstrating problem setup steps (i.e., reading the problem, making a diagram, stating assumptions, and connecting the problem to theory), as suggested in problem-solving technique references [9, 10, 12], to de-mystifying the appropriate mathematical framework for problem solving in chemical engineering, as depicted in the concept map. The strategy employed in the second year of the project was to emphasize, early in every problem-solving demonstration, the general equations (balances) to be solved, and then to guide the students in understanding and simplifying the problems through the lenses of the mathematics to be solved.

\subsection{Assessing Abilities and Confidence}

In the past two years, students were invited to fill out a survey to assess their problem-solving confidence level, perceptions, and abilities. At this point, data has been collected for two successive cohorts. Cohort 1 was assessed at the beginning and end of Course B (winter term of their second year of study), and again at the beginning of the fall term in their $3^{\text {rd }}$ year of study. Cohort 2 was assessed at the beginning of Course A (fall term of second year), and at the beginning of Course B (winter term of second year).

Data collection is on-going, and the sample set is insufficient to carry out detailed statistical analyses at this point because the first survey was collected midway through the first year of implementation, and data is not yet available on responses from students at the end of the second year of implementation. Survey responses were collected from 68 to 97 participants; Cohort 1 includes 104 students, and Cohort 2 includes 109 students. Results of these surveys are considered reliable and valid because at least $65 \%$ of students responded to each survey. Conversely, it must be noted that verbal feedback was collected informally and may not objectively reflect the views of all students. The same survey was administered each time [1]. In addition to rating their confidence level, students were asked to organize a list of problem-solving steps according to their perception of the easiest and the most difficult step:

1. Read the problem;

2. Connect problem to theory;

3. Make a diagram/picture of the problem;

4. State assumptions;

5. Develop mathematical equations;

6. Find values in tables/charts;

7. Perform computations;

8. Check numerical answer/unit consistency;

9. Other.

Results from this survey are combined with classroom observations and course evaluations to assess the effectiveness of the pedagogical approach and to adjust how the curriculum for Courses A and B is to be delivered in the following years.

\section{RESULTS AND DISCUSSION}

Data collections and observed trends are useful tools for instructors who seek to improve knowledge transfer and, in this case, specific problem-solving skills in junior-year chemical engineering students. However, tangible variations between cohorts must also be taken into consideration. For the two cohorts examined in this study, instructors note that the first cohort is especially fragmented with a large group of struggling students, and a cluster of very strong students, such that the marks distribution is bi-modal, with relatively few students obtaining marks near the class average. The second cohort is generally normally distributed, with the majority of students obtaining marks near the class average, although the overall marks distribution covers the entire range from below $40 \%$ to above $90 \%$ in both courses.

\subsection{Survey Results}

Generally, as was noted previously [1], students build on problem-solving skills first introduced to them during high school, and their first year of engineering education. In the survey, the first question asked is: "Do you feel confident in your problem-solving skills". Results for both cohorts are presented in Figure 3.

Preliminary results suggest that students from both cohorts exhibit the same confidence level at the start of Course B (inlet, winter of $2^{\text {nd }}$ year, shown in blue for Cohort 1, and in red for Cohort 2). Results for Cohort 1 indicate that student confidence level has generally increased by the end of the $2^{\text {nd }}$ year of study: proportions of students responding in the 1-2 confidence level range are lower and proportions in the 3-4 confidence level range are higher in the exit survey responses (shown in striped blue). However, this boost in confidence is short-lived and student responses to the survey administered at the inlet of 
$3^{\text {rd }}$ year are back to approximately the same levels as what was registered at the inlet to the winter semester of $2^{\text {nd }}$ year for Cohort 1. For Cohort 2, results suggest a slight drop in confidence level during the fall term of $2^{\text {nd }}$ year.

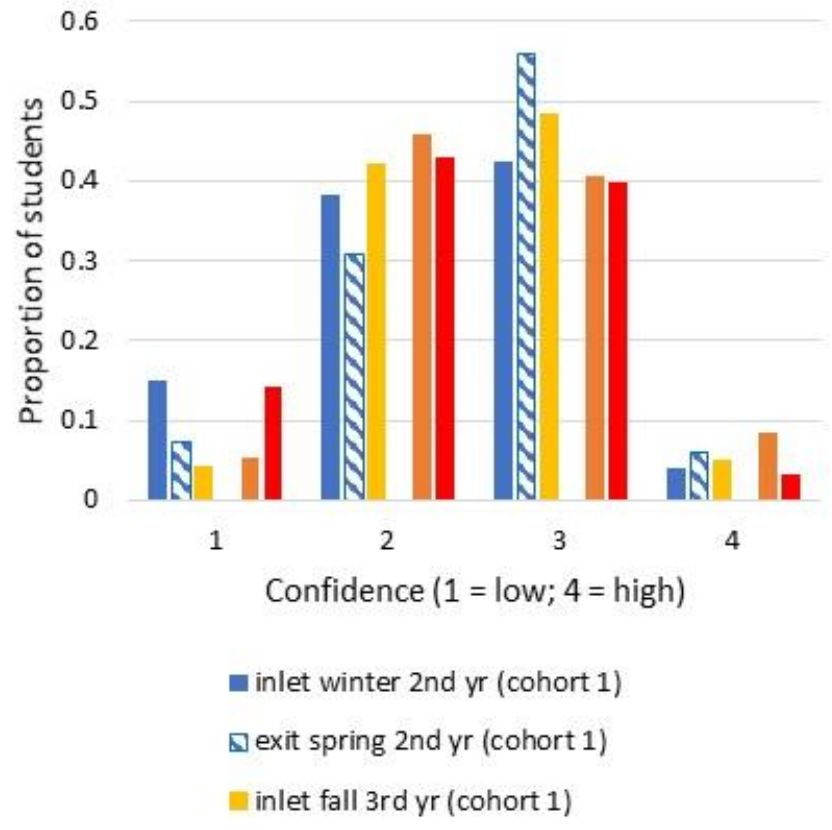

inlet fall 2nd yr (cohort 2)

- inlet winter 2nd yr (cohort 2)

Figure 3: Problem-Solving Confidence Level For each level, results for Cohort 1 are grouped in the set of three columns (blue, striped, yellow), and Cohort 2 results are shown in the last two columns (orange, red). An increased in confidence level was recorded for students surveyed at the end of Course B (exit, spring of $2^{\text {nd }}$ year), but this did not carry over to the start of the following term (inlet, fall of $3^{\text {rd }}$ year).

Despite the emphasis placed on learning how to solve problems using a prescribed methodology during the fall term, this drop in confidence level may be attributed to the drop in class average between the first and second years of engineering studies. Course A is considered a "gatekeeper" and students often perceive the material presented in this course to be especially challenging. Course A is a prerequisite to Course B as well as to several upper-year courses; mastering the contents of this course is pivotal to the future success of chemical engineering undergraduate students. Results in Course A are typically 5 to $7 \%$ lower than average marks obtained in first-year engineering courses, and approximately 10 to $18 \%$ of the class fails to achieve a passing grade in Course A.

The second part of the survey examines the perception of difficulty level for each problem-solving step. Results are summarized in Figure 4 (easiest step) and Figure 5 (most difficult step), where proportions of the student population are presented in histogram groups for each step. The response for step 9 ("other") was negligible to zero; thus, this step is not illustrated.

Students generally find that reading the problem, making a diagram, and performing computations are the easiest steps. With each successive response to the survey, as they progressed in their studies, a greater proportion of respondents also selected these as easiest steps. Therefore, it appears that the proposed problem-solving methodology may be helpful in guiding students through these steps. On the other hand, very few students selected step 5 (Develop mathematical equations) as an easy step. As illustrated in Figure 5, this step is by far the one considered most difficult by each cohort, regardless of when students responded to the survey, except for responses from the inlet of winter term for Cohort 2, where a greater proportion of students indicated that connecting the problem to theory (step 2) is most difficult. Examining responses regarding step 5, the proportions of students perceiving this step as most difficult drops between the start and end of each term (comparing the blue and striped columns and comparing the orange and red columns, respectively, in step 5 of Figure 5). It appears that students gain some confidence in their ability to develop mathematical equations during both Courses A and B. Nevertheless, as was noted from the responses in confidence levels, this boost is short-lived, and the proportion of students indicating that step 5 is the most difficult increased again for students entering their $3^{\text {rd }}$ year of study (step 5 of Figure 5, yellow column).

Overall, step 2 is considered the second most difficult aspect of problem solving. Furthermore, the proportions of students perceiving this step as difficult increase as students progress in their studies. This may be in response to tackling increasingly complex theory and problems as students advance in their studies.

In summary, as students progress through one course, their level of confidence generally increases; however, their central apprehensions when solving problems are consistent and persistent, i.e., developing the appropriate mathematical framework and relating the correct mathematical framework to theory are two elements where they feel something is lacking. The confidence boost is short lived. Moreover, and this may be a direct consequence of their perceived lack of acquired mathematical competency, their level of confidence drops when moving from one course to another. This calls for extending the current problem-solving project in theoretical courses beyond the second year to ensure continuity.

\subsection{Classroom Observations}

In problem-solving activities, notably in tutorials, students' understanding and confidence, once convinced of the central aspect of the mathematical formalism, were generally improved, at least for a given course. Another 
benefit observed in the classroom and reported in course evaluations is that students are also able to integrate abstract notions, notably in Course $\mathrm{B}$, and demonstrate their applications to real-world problems, at least to the level of competency sought for second-year chemical engineering students. The aspect of continuity between courses A and B is also valued as a positive experiment by students.

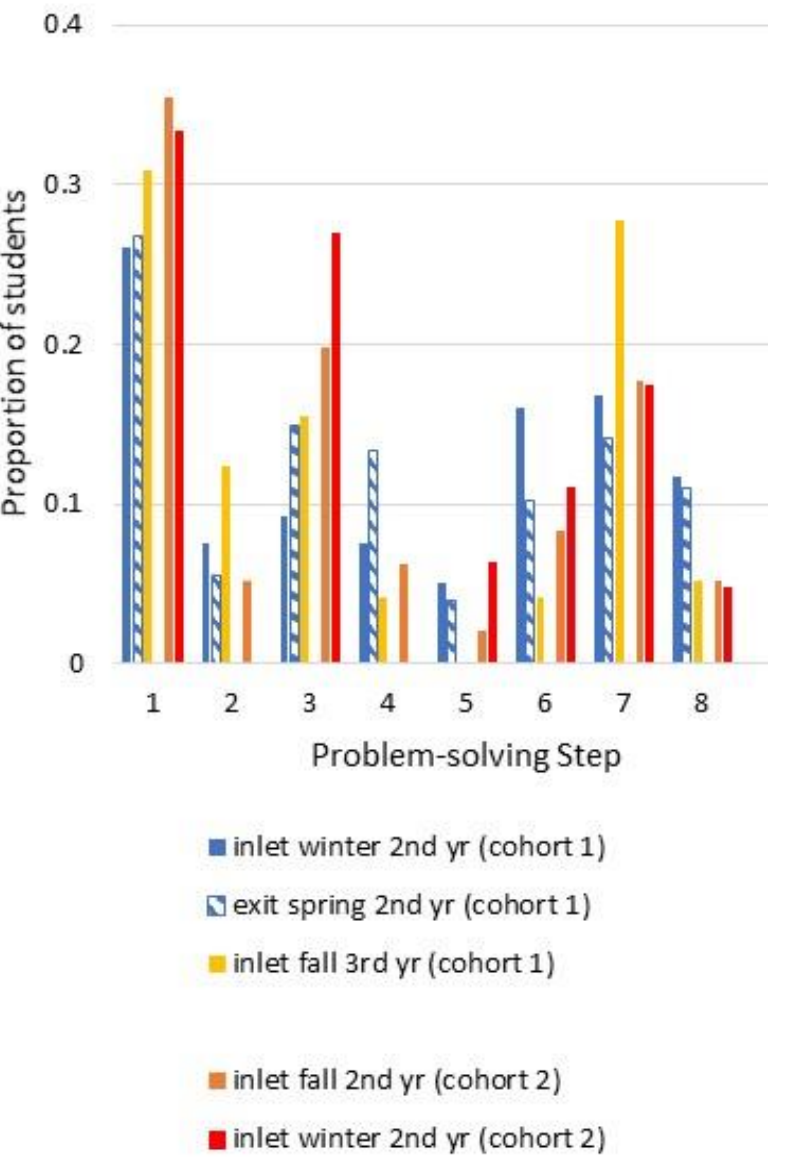

Figure 4: Easiest Problem-Solving Step

For each step, results for Cohort 1 are grouped in the set of three columns (blue, striped, yellow), and Cohort 2 results are shown in the last two columns (orange, red). Results vary between cohorts and as students progress through their studies. However, most students find that reading the problem (step 1), making a diagram (step 3), and performing computations (step 7) are relatively easy steps.

As Courses A and B progress, an increase in students' confidence measured through surveys can be observed for most students. Informal feedback mirrors this result. The fact that these courses propose a common problem-solving methodology is generally viewed by the students as a positive endeavour. But the overall understanding of chemical engineering problems, as depicted Figure 2, does not fully permeate the students' mind at this early stage of their chemical engineering studies. Next, we present observations about students' behaviour in each class and propose new teaching approaches to promote the proposed problem-solving methodology.

beginning of Course A. The thought process required to implement the proposed problem-solving methodology is described in detail through the first tutorials, during which students are invited to incrementally and interactively solve relatively simple mass balance problems, while reflecting on the process itself.

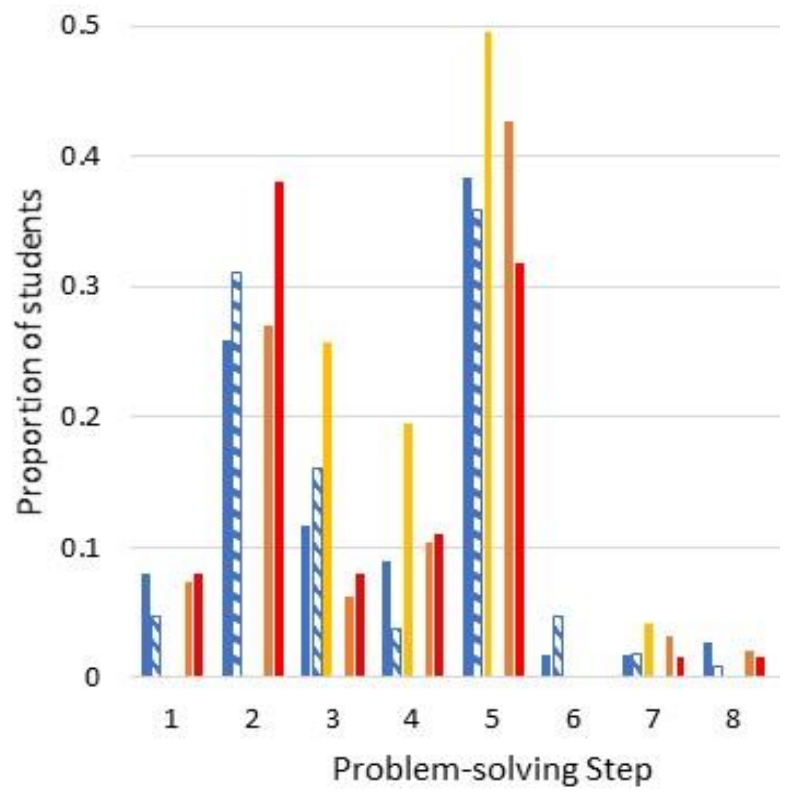

$$
\begin{aligned}
& \text { = inlet winter 2nd yr (cohort 1) } \\
& \text { D exit spring 2nd yr (cohort 1) } \\
& =\text { inlet fall 3rd yr (cohort 1) }
\end{aligned}
$$

$$
\begin{aligned}
& \text { inlet fall 2nd yr (cohort 2) } \\
& \text { [ inlet winter 2nd yr (cohort 2) }
\end{aligned}
$$

Figure 5: Most Difficult Problem-Solving Step For each step, results for Cohort 1 are grouped in the set of three columns (blue, striped, yellow), and Cohort 2 results are shown in the last two columns (orange, red). Results vary between cohorts and as students progress through their studies. However, most students find that connecting the problem to theory (step 2), and especially developing mathematical equations (step 5) are the most difficult steps.

Students are first introduced to the concept map at the

Demonstrations of solved problems focus on the questions that must be asked by the problem solver to connect the theory to the problem and to implement the appropriate equations that express in mathematical form 
the physical reality of the problem. Students are invited to ask each other to explain their problem-solving thought process as they solve a problem. This approach is systematically presented for each of the numerous problems solved in class during Course A to solve mass and energy balance problems. Making the students aware of the process of acquiring new knowledge as they practice problem solving may be helpful in anchoring the concepts and in developing strong problem-solving habits.

In the first week of Course $B$, expectations of problem-solving are laid out in detail, and students are made aware that theory (First and Second laws of thermodynamics) is to be presented and tested solely through solutions of balances. Every problem, for closed and open systems alike, is designed around mass, energy, and entropy balances, to the extent that some students find the presentation repetitive. Each week, classes include roughly five fully developed examples, two video examples, one tutorial, and two homework problems to be solved following the prescribed problem-solving methodology. The drawback is that, once they have accepted the methodology, students may not feel the need to reflect on their own problem-solving methodology. In other words, some students might passively apply the methodology within the realm of Course B set of problems without accepting the proposed methodology as a life-long approach to problem-solving. In future years, based on these observations, Course B is to evolve such that students become more active in the classroom, and adopt a comprehensive and systematic methodology as their own.

\section{CONCLUSIONS}

This contribution offers new evidences that a common approach to teach students problem solving is valuable at an early stage of their engineering studies. This contribution also builds on the theory originally presented [1] by fostering, through objectivism, cognition, and constructivism, the development of long-lasting habits and confidence in students' problem-solving abilities. Raising the awareness of the process of learning is also important for students to successfully incorporate new knowledge. In the next stage of this projects, instructors of Courses A and B will seek to extend the common approach beyond second year, by getting upper-year instructors involved. Surveys and requests for feedback will be expanded to obtain further details on students' perceptions of their learning needs and developing abilities; as more data is collected, statistical analyses will be performed to characterize longterm trends in this study. Finally, new teaching activities and approaches will be considered in the delivery of material to foster students' ownership of an effective and consistent problem-solving methodology, i.e., by guiding students in the development of long-lasting problemsolving methodology beyond the second year.

\section{Acknowledgements}

The authors wish to thank N. Graham, who produced graphics in support of this article. The authors acknowledge funding support from the National Science and Engineering Research Council (Discovery grants for the first author) and from the Faculty of Engineering and Applied Science at Queen's University.

\section{References}

[1] Meunier, L. and Hudon, N. 2019. "Translating Problem Statements into Actionable Solution Procedure: Developing Problem-Solving Skills in Chemical Engineering Second-Year Students". in Proc. CEEA Canadian Engineering Education Conf. (Ottawa, ON; 8-12 June), 8 pp. 2019.

[2] Narayan, R., Rodriguez, C., Araujo, J., Shaglaih, A., and Moss, G. 2013. "Constructivism - Constructivist Learning Theory", in B.J. Irby, G. Brown, R. LaraAlecio, and S. Jackson (Eds): The Handbook of Educational Theories. Information Age Publishing, USA, 169-183.

[3] Hoover, W.A. 1996. The Practice Implications of Constructivism. SEDL Letter, IX(3).

[4] Jonassen, D.H. (1991). "Evaluating Constructivistic Learning", Educational Technology, 31(9), 28-33.

[5] Bates, A.W. 2019. Teaching in a Digital Age; Guidelines for designing teaching and learning. Open Book by BC Campus - BC Open Textbooks Adapted and Created by BC Faculty. Available from https://opentextbc.ca/teachinginadigitalage/

[6] Cronjé, J. 2006. "Paradigms Regained: Toward Integrating Objectivism and Constructivism in Instructional Design and the Learning Sciences", Educational Technology Research and Development, 54(4), 387-416.

[7] Jenlink, P.M. 2013. "Situated Cognition Theory", in B.J. Irby, G. Brown, R. Lara-Alecio, and S. Jackson (Eds): The Handbook of Educational Theories. Information Age Publishing, USA, 185-198.

[8] Dewey, J. 1938. Experience and Education. Kappa Delta Pi Lecture Series. New York, NY, Collier Books, $96 \mathrm{p}$.

[9] Wankat, P.C. and Oreovicz. F.S. 2015. Teaching Engineering, 2nd Ed. Purdue University Press, USA. $450 \mathrm{p}$.

[10] Polya, G. 2004. How to Solve It. Princeton University Press. 253 p.

[11] Pretz, J.E., Naples, A.J. and Sternberg, R.J. 2003. "Recognizing, Defining, and Representing Problems", in J.E. Davidson and R.J. Sternberg (Eds): The Psychology of Problem Solving. Cambridge University Press, UK, 1-30.

[12] Wood, D.R., 2000. An Evidence-Based Strategy for Problem Solving. Journal of Engineering Education, 89(4), 443-459. 